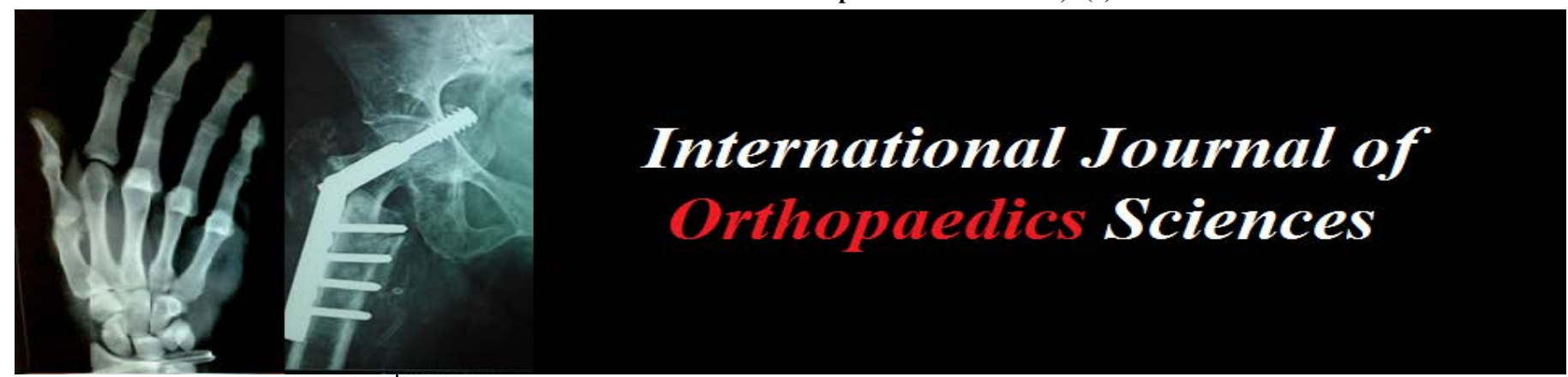

E-ISSN: 2395-1958

P-ISSN: 2706-6630

IJOS 2021; 7(4): 263-267

(C) 2021 IJOS

www.orthopaper.com

Received: 23-07-2021

Accepted: 26-08-2021

Jaydeep K Patil

Assistant Professor,

Department of Orthopaedics,

Prakash Institute of Medical

Sciences and Research, Islampur,

Maharashtra, India

Dr. Pramod G Kuchekar

Associate Professor,

Department of Orthopaedics,

Prakash Institute of Medical

Sciences and Research, Islampur,

Maharashtra, India
Corresponding Author:

Dr. Pramod G Kuchekar

Associate Professor,

Department of Orthopaedics,

Prakash Institute of Medical

Sciences and Research, Islampur,

Maharashtra, India

\section{A prospective study of functional and radiological outcome of open reduction and internal fixation of malleolar fracture and associated complications}

\author{
Jaydeep K Patil and Dr. Pramod G Kuchekar
}

DOI: https://doi.org/10.22271/ortho.2021.v7.i4d.2894

\section{Abstract}

Background: Malleolar fractures are a kind of regularly seen fractures in orthopedic department. As with all intra articular fractures, malleolar fractures necessitate anatomical reduction and stable internal fixation.

Objective: To study functional and radiological outcome of open reduction and internal fixation of malleolar fracture and associated complications and to study role of early mobilization and graded weight bearing.

Materials and Method: This study prospectively evaluated clinical, radiological and functional outcome of 39 patients of malleolar fracture treated with open reduction and internal fixation at Madras institute of orthopaedics and traumatology, Chennai from May 2016 to May 2017. After open reduction, patients were followed clinically and radio logically at 6 weeks, 3 months and 6 months. After operative procedure, functional and radiological outcome was assessed using Baird and Jackson score system.

Results: Majority were Supination-External Rotation injuries 19 cases. Most common implant used for medial malleolus fracture was 3.5mm cortical screw (84.6\%) cases and for lateral malleolus semi tubular plate (64.1\%). According to Baird and Jackson scoring system our results were excellent in 6 cases (15.4\%), good in 22 cases (56.4\%), fair in 8 cases (20.5\%), poor in 3 cases (7.7\%). Good results were observed in maximum patients, one patient developed infection and two patients developed ankle stiffness.

Conclusion: Open reduction and internal fixation of malleolar fracture is necessary to achieve anatomical reduction of the fracture fragments, ankle mortise congruity, restoration of the length of the fibula and restoration of syndesmotic integrity. We recommend to apply POP cast to prevent equines deformity and to start early range of motion of ankle as early as possible in cast after making dorsal window cut for 1 month.

Keywords: Open reduction, internal fixation, malleolar fracture, outcome, Baird and Jackson scoring

\section{Introduction}

Ankle injuries are usually mixed injuries, ligamentous and bony; each injury is a consequence of the failed ligament and bony parts owing to deformation. Malleolar fractures present a diverse picture leading to broad classification systems, of which three are in vogue, LaugeHansens classification, Dennis-Weber classification and AO classification [1]. Malleolar fractures are one of the most common fractures in orthopaedic traumatology. Malleolar fractures warrant accurate reduction and stable internal fixation. When malleolar fractures are not reduced accurately, they may lead to post traumatic painful restriction of motion or early osteoarthritis or both. Unstable, displaced and open fractures are treated using open reduction and internal fixation ${ }^{[2]}$. The superiority of Open Reduction and Internal Fixation over closed treatment has been thoroughly demonstrated in literature. Different studies have come with different results in case of malleolar fractures management approach ${ }^{[3]}$. The purpose of this study was to assess the functional and radiological outcome after performing open reduction and internal fixation of malleolar fractures. The purpose of this study was to study functional and radiological outcome of open reduction and internal fixation of malleolar fracture and associated complications and to study role of early mobilization and graded weight bearing. 


\section{Material and Methods}

This was a prospective observational study with a sample size of 39 patients, in age-group 18 to 65 years, with malleolar fractures of ankle presenting to Madras institute of orthopaedics and traumatology, Chennai during 2016 and 2017. Inclusion criteria was age 18 years to 65 years with unstable malleolar fractures, all closed type of malleolar fractures of ankle and type I and type II compound malleolar fractures of ankle. Exclusion criteria removed cases of compound malleolar fractures of type III A, B and C, all malleolar fractures associated with pilon or plafond fractures of tibia, Previous ankle fracture, multiple trauma, associated with bilateral lower limb fractures, any concomitant painful or disabling disease of lower limb that could interfere with evaluation of the affected ankle, and patient who did not follow up regularly and for at least 6 months and any other fracture that limited the mobilization.

On admission of the patient, a careful history was elicited from the patient or attendants to reveal the mechanism of injury and the severity of the trauma. The cases were then clinically evaluated to determine their overall condition and local injury. Vital signs and comprehensive systemic examinations were done to assess the patient's condition. Methodical examination was done to rule out fractures at another site.

\section{Management}

Swelling, deformity and loss of function were noted on local examination. Tenderness and crepitus at the fracture site were elicited on palpation. The pulsations of the posterior tibial and dorsalis pedis arteries, capillary filling, local temperature, pallor, and paranesthesia were used to determine the distal neurovascular state. The patients' hemodynamics were stabilized, and appropriate analgesia was provided with Inj Tramadol or Inj Diclofenac. Anteroposterior and lateral radiographs of the affected leg were taken and fracture patterns were classified based on Lauge-Hansen classification. Patients were fitted with a below-knee splint and a posterior plaster of Paris slab. Leg elevation cot end elevation and ice application.

\section{Preoperative steps}

After the radiological evaluation, patients were informed the need for operative approach of treatment. General Physicians assessed the patient's comorbid conditions, and further by anesthesiologists. Next, informed consent was obtained for operative procedure. Second generation Cephalosporins were administered at the time of induction of anesthesia.

\section{Operative Procedure}

Spinal anesthesia was administered to 36 patients and General anesthesia for 3 patients. In cases of lateral malleolar fracture, the patient was positioned in a supine position with a sandbag under the ipsilateral buttock. The tourniquet was inflated after the exsanguinations, and the time was recorded. The affected limb was prepared with scrub with chlorhexidine solution. Surgical draping was done and the foot was covered with a hand towel or a glove. Depending on the style of fixation planned, the operative approach for the fixation of the lateral malleolus was done with standard practices. Firstly, lateral malleolus was exposed and fixation was done in 35 cases. As per standard procedures, medial malleolus fixation was done in 37 patients. Almost all cases we found that there was soft tissue interposition between fracture fragments. Posterior malleolar fracture was noted in 17 cases. The posterior malleolar fracture was fixed for 8 patients and other patient has got anatomical reduction. 29 patients were operated within 24 hours of the injury. 10 patients were operated between two and five days because of uncontrolled Diabetes and due to poor soft tissues. Tourniquet control was standard for all patients undergoing procedure. The duration of surgery varied from 30 mins to 60 minutes.

\section{Implants}

The medial malleolus was fixed with 3.5 cortical screw in 33 cases, Tension band wiring in 2 cases and semi tubular plate in 2 cases. The lateral malleolus was fixed with Semi tubular plate in 25, Tension band wire in 2 and Dynamic compression plate in 8. In 2 cases, Syndesmotic screw were used. Normal saline was used to wash wound and 2-0 Vicryl were used for sutures. Skin was closed with 3-0 Ethilon or Staple. Dressing with sufficient padding and a below knee plaster of Paris was put.

\section{Postoperative}

Patients were administered adequate analgesics and IV Antibiotics for 5 days postoperatively. Foot elevation achieved with pillows. Radiological assessment was done with AP and lateral views. First dressing done on the second postoperative day. Patient was mobilized with walker on the first post-op day without bearing weight on affected limb. On $3^{\text {rd }}$ postoperative day POP CAST was applied, 4th postoperative day dorsal window cutting made for ankle mobilization. Patients were discharged on the fifth day on an average.

\section{Follow-up}

The patients were followed up at $4^{\text {th }}$ week, $6^{\text {th }}$ week, $3^{\text {rd }}$ Month and $6^{\text {th }}$ month. POP cast and sutures were removed at follow up in the outpatient clinic at the end of 4th weeks and crepe bandage applied. Check $\mathrm{x}$ rays were done. Signs of healing and status of the joint was noted. Partial weight bearing was started with support. X-ray results were used to decide on full weight bearing. Patients were followed up at 6 weeks, 3 months, 6 months and patients were evaluated as per the rating of the Baird and Jackson criteria. This study was conducted after obtaining clearance from institutional ethical clearance and informed consent. Statistical analysis was done in Microsoft Excel software and results are presented in frequencies and percentages.

\section{Results}

Out of the total 39 participants, majority categories were male (61.5\%), 21-40 years age group (74.4\%), Right side Malleolar fractures (53.8\%), Road traffic accidents (59\%), Supination external rotation (48.7\%), simple fractures (89.7\%), trimalleolar fracture (43.6\%). The functional outcome was good in 56.4\%, excellent in $15.4 \%$, and fair in $20.5 \%$. Functional outcome was poor in $7.7 \%$ participants. Table1 shows distribution of study participants by sociodemographic factors and other parameters $(\mathrm{N}=39)$. 
Table 1: Table shows distribution of study participants by sociodemographic factors and other parameters. (N=39)

\begin{tabular}{|c|c|c|c|c|c|}
\hline Parameter & Number (n) & $\%$ & Parameter & Number (n) & $\%$ \\
\hline Age group (years) & & & Type of Fracture & & \\
\hline $21-30$ & 17 & 43.6 & Simple & 35 & 89.7 \\
\hline $31-40$ & 12 & 30.8 & Compound & 04 & 10.3 \\
\hline $41-50$ & 5 & 12.8 & Type of fracture: Medial Malleolus & 4 & 10.3 \\
\hline $51-60$ & 4 & 10.3 & Lateral Malleolus & 2 & 5.1 \\
\hline $61-70$ & 1 & 2.6 & Bimalleolar & 16 & \\
\hline \multicolumn{6}{|c|}{ Gender } \\
\hline Males & 24 & 61.5 & Trimalleolar & 17 & 43.6 \\
\hline Females & 15 & 38.5 & Complication Infection & 1 & 2.6 \\
\hline \multicolumn{6}{|c|}{ Malleolar Fracture } \\
\hline Right side & 20 & 53.8 & $\begin{array}{l}\text { Ankle stiffness } \\
\end{array}$ & 2 & 5.1 \\
\hline Left side & 19 & 46.2 & No complication & 36 & 92.3 \\
\hline Mode of injury & & & Functional outcome & & \\
\hline RTA & 23 & 59.0 & Excellent & 06 & 15.4 \\
\hline Domestic Fall & 16 & 41.0 & Good & 22 & 56.4 \\
\hline \multicolumn{6}{|c|}{ Mechanism of injury as per Lauge - Hansen classification } \\
\hline SA & 5 & 12.8 & Fair & 8 & 20.5 \\
\hline SER & 19 & 48.7 & Poor & 3 & 7.7 \\
\hline PA & 9 & 23.1 & & & \\
\hline PER & 6 & 15.4 & & & \\
\hline
\end{tabular}

Pics
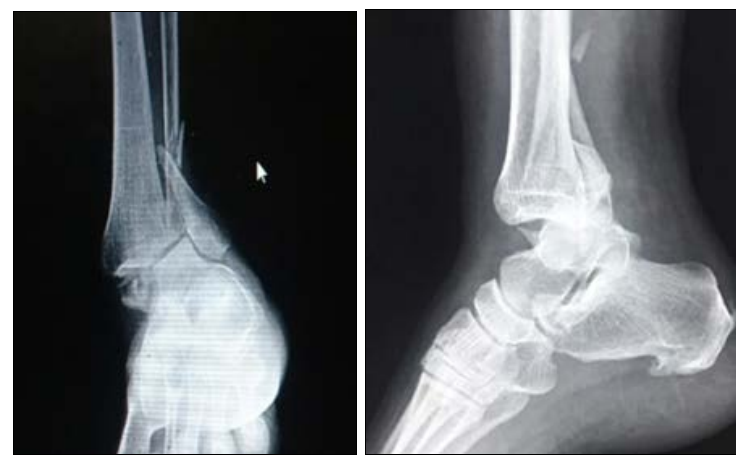

Fig 1: Showing case 3 Pre-op ray with bimalleolar ankle fracture dislocation

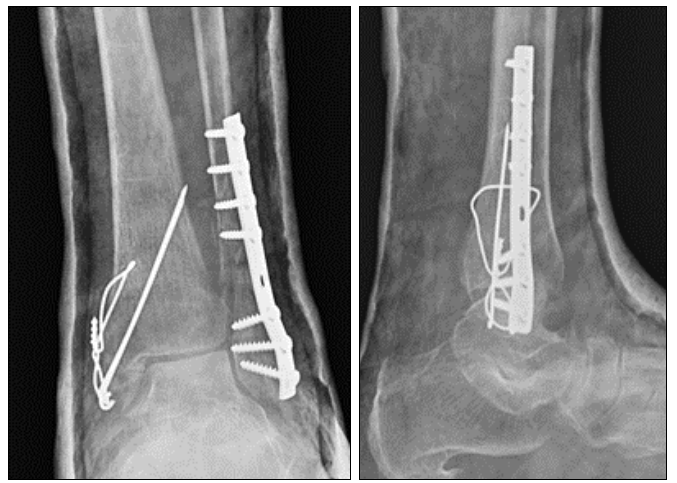

Fig 2: Showing Case 3 Postoperative $\mathrm{x}$-rays after 1month

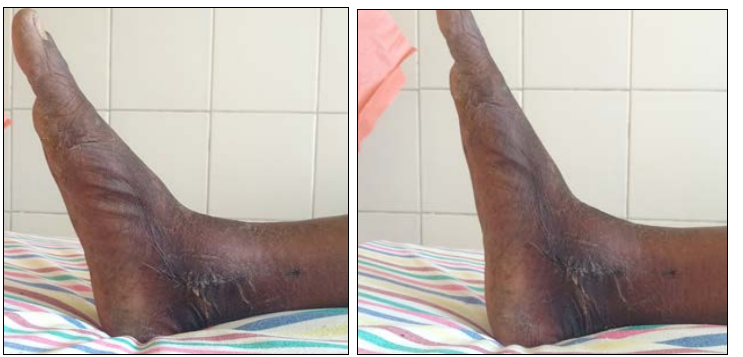

Fig 3: Showing case 3 ankle dorsiflexion and plantar flexion (Baird \& Jackson's score at 6 month was 58)

\section{Discussion}

Increased knowledge about the normal and post traumatic anatomy and function of the ankle joint, has created opportunity for exact reduction and tough fixation of the ankle fractures. Prompt operative treatment of the displaced ankle fractures decreases morbidity and improves functional outcome. Accurate open reduction and stable internal fixation using AO method and principle for malleolar fractures gave a high percentage of excellent and good results ${ }^{[4]}$. This study supports these conclusions. Although the scoring of Baird and Jackson ${ }^{[5]}$ has proven to be strict allowing only very small fluctuation from normal, about 6(15.4\%) patients in this series achieved excellent,22(56.4\%) patients good, 8(20.5\%) patients fair and $3(7.7 \%)$ patients poor results by this scoring system and also had perfect anatomical reduction.

Percentage of simple fractures, and mode of injury (road traffic accidents) and supination external rotation (as per Lauge-Hansens classification system) in our study was similar to the one reported in several other studies ${ }^{[4-7]}$. In our study, at final follow up, we obtained $28(71.8 \%)$ patients good to excellent results as per Baird and Jackson ${ }^{[5]}$ scoring system, which is less than other studies most probably because we limited our follow up period for 24 weeks only and very few patients could run. In all other studies, the follow up period varied between 5 to 12 years and all patients running ability was evaluated at final follow up. Another study with 40 cases of bimalleolar fractures of ankle treated by ORIF, showed excellent results in $57.5 \%$, good in $25 \%$, fair in $12.5 \%$, and poor in $5 \%{ }^{[8]}$. A similar study of 21 patients treated, overall good to excellent results were obtained in $80.1 \%{ }^{[9]}$. Our findings were comparable to these two studies and Maruti et al. study ${ }^{[10]}$. In Colton et al. study, $70 \%$ showed good to excellent results ${ }^{[11]}$. Where as in a large study with 132 patients showed that $77.3 \%$ had good outcome and $16 \%$ with fair results and poor in $6 \%{ }^{[12]}$. DeSouza et al. got good results in $90 \%$ participants ${ }^{[13]}$.

The mechanical stress pattern occurring in the ankle after open reduction and internal fixation is not necessarily identical to the injury process. Furthermore, in vertical fractures it is advisable to provide maximal stress resistance against the two mechanical forces on the medial side (varus stress, axial compression) regardless of initial injury mechanism. The vertical medial fracture pattern, tend to 
migrate proximally even if fixed with lag screws. These vertically oriented medial malleolar fractures are occasionally associated with marginal impaction of the medial articular cartilage. Dis-impaction and restoration of the articular surface leave a metaphysical defect that compromises the stability of the lag screws. Therefore, if vertical fracture of medial malleolus was encountered in our study, we applied buttress plate on medial side and lag screw applied through the plate.

Observation in this study support the contention of Yablon et al. ${ }^{[14]}$ that lateral malleolus is key to anatomical reduction of the malleolar fractures, because the displacement of the talus faithfully followed that of the lateral malleolus. Persistent lateral displacement or residual shortening would arise from poor reduction of the distal section of the fibula. This does not necessarily lessen the importance of the medial malleolus in contributing to the congruity of the medial aspect of the ankle, but it serves to emphasize that the lateral malleolus should no longer be ignored in the treatment of ankle injuries. The post traumatic arthritis prognosis correlates with the size of the posterior malleolar fragment, with large fragment (those involving area more than $25 \%$ of the tibial articular surface) having unsatisfactory outcomes. However, posterior syndesmotic ligaments are invariably attached to a small avulsion fragment leading to micro-instability of tibiotalar joint and early post traumatic articular degeneration. 17 patients in our study had posterior malleolar fracture, out of these 8 patients were treated by fixing the posterior malleolus all other fracture had small fragment less than 25\% articular surface or in some cases it falls back with fixation of medial and lateral malleolus. In our study at the final follow up 6 patients showed excellent outcome, 22 showed good, 8 showed fair and 3 patients had poor result, most probably due to micro instability of the tibiotalar joint.

A number of different treatment regimens have been suggested postoperatively. Burnwell \& charnley ${ }^{[7]}$ advocated postoperative joint mobility exercises in bed until motion was restored followed by full weight bearing in a cast. LundKristensen et al. either used no cast or applied one for a few days postoperatively and then allowed full joint mobilization out of the cast. They advocated the use of crutches to maintain a non-weight bearing status. Meyer and Kumler used a postoperative cast but only for an average of 3.8 weeks followed by non-weight bearing mobilization until fracture union. According to Makwana $\mathrm{NK}^{[15]}$ the risk of complications after internal fixation is low but higher with closed treatment. Most of the complications were minor which resolved within 3 months. In this study there were no non-union of the medial malleolus and no malunion, which were reported in some series, where $30 \%$ - $48 \%$ patients developed nonunion and malunion after closed reduction.

Our study suggests that a ruptured deltoid ligament can be left unexplored, thereby reducing the soft tissue manipulation and surgery time. All ankle fractures after fixation treated by early mobilization and gradually increased weight bearing. Protected mobilization in slab or cast with dorsal window for 4 weeks allows soft tissues to heal adequately and, in our study, only one patient showed clinical instability at final visit. The number of patients with posterior malleolus fracture who had residual displacement was relatively small, but the results confirmed the general impression that such injuries are of little significance when they involve less than $25 \%$ of the surface. Plaster cast immobilization, for initial 4 weeks, did not diminish ankle motion. The ranges of motion were reduced initially but after cast removal ankle movements rapidly improved. The rapid gaining of motion from 6 to 12 weeks may be due to the positive attitude to exercise, psychologically due to relief from cast and resumption of weight bearing at six weeks. Malleolar fractures of ankle have a varied presentation. They can range from isolated fibular fractures with no displacement to a trimalleolar fracture with dislocation and vascular compromise. A broad understanding of all aspects of mechanism of injury, pathologic anatomy and treatment options coupled with training experience is required before any attempt should be made to treat these injuries with thorough understanding of injury patterns repair of the damaged ankle joint can lead to rewarding outcomes for the patient and physician. Perfect Anatomical reduction and good internal fixation, post-operative ankle mobilization is directly proportional to the final result. Limitations of the present study are that 6 months follow up is inadequate, longer follow up are required to evaluate the post-operative ankle arthritis and functional outcome. Larger number of sample size would have been better to evaluate functional outcome.

Our study recommendations are open reduction and internal fixation of malleolar fracture is necessary to achieve anatomical reduction of the fracture fragments, ankle mortise congruity, restoration of the length of the fibula and restoration of syndesmotic integrity. If patient comes with ankle fracture dislocation, then immediately, we have to reduce and keep into slab, if unstable then we have to put intraarticular k-wire and slab application. Fracture must be fixed as early as possible preferably within 24 hours to prevent post-operative complications like excessive swelling, superficial infection and to start early mobilization. Percutaneous fixation of medial malleolus should be avoided and open reduction and internal fixation should be done. As soft tissue interposition between fractures may lead to delayed union or nonunion. We recommend the application of a posterior plaster of Paris slab post operatively and convert into cast application after subsides of swelling and dorsal window cutting and start range of motion of ankle. So, it prevents swelling and equines deformity as well as to aid wound healing. We recommend to start range of motion of ankle as early as possible in cast dorsal window for 1 month and after 1 partial weight bearing around 4 weeks to prevent post- operative ankle stiffness. Uncontrolled diabetes should be controlled to prevent post- operative wound related complications.

\section{Source of Funding: None}

\section{Conflict of interest: None}

\section{References}

1. Tartaglione JP, Rosenbaum AJ, Abousayed M, DiPreta JA. Classifications in Brief: Lauge-Hansen Classification of Ankle Fractures. Clin Orthop Relat Res 2015;473(10):s3323-3328. PMCID: PMC4562928.

2. Verhage SM, Boot F, Schipper IB, Hoogendoorn JM. Open reduction and internal fixation of posterior malleolar fractures using the posterolateral approach. Bone Joint J 2016;98-B(6):812-7. PMID: 27235525.

3. Colaco RM, Gudda GNL, Hanumant WA, Mahesh SG, Faisal Q, Badgire K. Functional outcome of surgical management of malleolar fractures of the ankle joint using BairdJackson scoring - A Prospective study. Int. J med. health res 2017:3(1);42-45.

4. Surgical treatment of malleolar fractures-a review of 144 patients. Beris AE, Kabbani KT, Xenakis TA, Mitsionis 
G, Soucacos PN. Clinical Orthopaedic Related Research 1997;341:90-98.

5. Fracture of the distal part of fibula with associated disruption of deltoid ligament. Baird RA and Jackson ST; JBJS 1987;69A:1346-52.

6. Surgical treatment of displaced ankle fractures. Roberts RS; Clinical Orthopaedics 1983;172;164-170.

7. The treatment of displaced fractures at the ankle by rigid internal fixation and early joint movement. Burnwell HD \& Charnley AD; JBJS 1965;47B:643-660.

8. Mohan $\mathrm{K}$ et al. To assess the clinical outcome of ankle fractures with regards to the demographic variables and the quality of reduction of the fractures. International Journal of Orthopaedics Sciences 2017;3(2):445-448.

9. Shams N, Ahmed I, Hegde A. A study on surgical treatment of ankle fractures- A clinical study of 21 cases. IJBAR 2014;05(04):190-92.

10. Maruthi CV, Venugopal N, Nanjundappa HC, Dr.Siddalinga S. Bimalleolar Fracture of Ankle Joint Managed By Tension Band Wiring Technique: A Prospective Study. Sch. J App. Med. Sci 2014;2(1D):428-432.

11. Burwell HN, Charnley AD. The treatment of displaced fractures at the ankle by rigid internal fixation and early joint movement. J Bone Joint Surg 1965;47B:634-660.

12. Colton CL. The treatment of Dupuytren's fracture dislocation of the ankle. J Bone Joint Surg 1971;53B:6371.

13. Desouza LJ, Gustilo RB, Meyer TJ. Results of operative treatment of displaced external rotation-abduction fractures of the ankle. J Bone Joint Surg 1985;67A:10661074.

14. The key role of the lateral malleolus in displaced fractures of ankle. Yablon IG, Heller FG, Shouse L. JBJS 1977;57A:169-173.

15. Makwana NK, Bhowal B, Harper WM, Hui AW. Conservative versus operative treatment for displaced ankle fractures in patients over 55 years of age. A prospective, randomized study. J Bone Joint Surgery $\mathrm{Br}$ 2001;83(4):525-9. 